\title{
Late Ordovician Palynomorphs
}

\author{
S. G. MOLYNEUX ${ }^{*}$ \& F. PARIS ${ }^{2}$ \\ ${ }^{1}$ British Geological Survey, Keyworth, Nottingham NG12 5GG, U.K. \\ ${ }^{2}$ Laboratoire de Paléontologie et de Stratigraphie, Université de Rennes, 35042 Rennes Cedex. France.
}

\section{ACRITARCHS}

Ordovician acritarchs have been recorded in five core samples collected between $2520 \mathrm{ft}$. and $3000 \mathrm{ft}$. in Well E1-81, and ten cutting samples taken between $12150 \mathrm{ft}$. and $13240 \mathrm{ft}$. in Well J1-81A. All the assemblages recovered are of Late Ordovician age; no Early Ordovician or Middle Ordovician assemblages have been identified.

Investigations have so far concentrated on the acritarch assemblages from Well E1-81. The highest three Ordovician samples from depths of 2520 to $2550 \mathrm{ft}$., 2552 to $2557 \mathrm{ft}$., and 2562 to $2567 \mathrm{ft}$., yielded similar assemblages which include Veryhachium irroratum, $V$. cf. lairdii, $V$. oklahomense?, V. subglobosum, V. trispinosum, Villosacapsula setosapellicula and a new species, Striatotheca sp. A. Navifusa similis? is represented by one specimen in the sample from 2552 to $2557 \mathrm{ft}$. Another specimen from the same sample is tentatively referred to Aremoricanium syringosagis. Specimens of Baltisphaeridium, Peteinosphaeridium, Leiofusa and Eupoikilofusa occur throughout the interval 2520 to $2567 \mathrm{ft}$. but are rare. Commonly occurring species include $V$. irroratum and $V$. setosapellicula. $V$. irroratum has been recorded from the Middle Ordovician of North America (Loeblich \& Tappan, 1969) and the Caradoc of England (Turner, 1984) but Cramer \& Diez (1979) maintain that it has its acme in the Ashgill. $V$. setosapellicula is common in the Sylvan Shale of Oklahoma (Loeblich, 1970) which is generally understood to be of Ashgill age, but is rare in the Eden Shale (Caradoc) of Indiana (Colbath, 1979) and in the type section of the Caradoc Series in Shropshire, England (Turner, 1984). The common occurence of these two species could be taken to indicate an Ashgill age for the interval between 2520 and $2567 \mathrm{ft}$., an age which is consistent with the chitinozoan evidence for the age of samples from $2572 \mathrm{ft}$., and 2558 to $2574 \mathrm{ft}$. (see below).

A major change in the composition of the acritarch assemblages takes place between $2567 \mathrm{ft}$. and $2790 \mathrm{ft}$. in Well E1-81. A number of the species that are recorded above this interval also occur in samples from 2790 to $2840 \mathrm{ft}$. and 2970 to $3000 \mathrm{ft}$. These include $V$. irroratum, $V$. cf. lairdii, $V$. oklahomense?, $V$. subglobosum, $V$. trispinosum and $V$. setosapellicula.

${ }^{*}$ Published with the approval of the Director, British Geological Survey (N.E.R.C.).
Striatotheca sp. A, however, is absent below $2790 \mathrm{ft}$., $V$. irroratum and $V$. setosapellicula are less common, and Veryhachium cf. reductum is present. Other species present below $2790 \mathrm{ft}$, include Actinotodissus cf. crassus, Baltisphaeridium longispinosum delicatum, ?Baltisphaerosum bystrentos, B. christoferii, Dactylofusa spinata?, Goniosphaeridium cf. conjunctum, Ordovicidium heteromorphicum?, Orthosphaeridium cf. chondrododora and Peteinosphaeridium nudum. Several of these species occur in the type Caradoc Series in England (Turner, 1984), in the Eden Shale (Caradoc) of North America (Loeblich \& Tappan, 1978; Colbath, 1979) and in the Viruan (Middle Ordovician) of Sweden (Kjellstrom, 1971). They suggest a Caradoc, possibly late Caradoc, age for the sediments between $2790 \mathrm{ft}$. and $3000 \mathrm{ft}$.

\section{CHITINOZOANS}

Recent work on Late Ordovician chitinozoan assemblages from Anticosti Island (Canada), the Baltoscandian area and the Anti-Atlas region of Morocco has added substantially to the body of available data on the distribution of Ordovician chitinozoans, enabling relatively precise age determinations to be proposed for the assemblages recovered in the present study.

Neither Early Ordovician nor Middle Ordovician assemblages have been identified in the material so far investigated. Two wells, E1-81 (core samples from depths 2558 to $2574 \mathrm{ft}$. and $2572 \mathrm{ft}$.) and J1-81 A (eleven cutting samples ranging from depth 12150 to $13337 \mathrm{ft}$., the deepest of which from 13240 to $13337 \mathrm{ft}$., being barren) have yielded Ashgill assemblages. The main species recorded are: $C$. (Calpichitina) lenticularis, Armoricochitina nigerica, Plectochitina sylvanica, Tanuchitina bergstroemi and Ancyrochitina merga (or closely related forms). Armoricochitina nigerica is the most abundant species in the assemblages from Well E1-81, which are less diverse in composition than the assemblages from Well J1-81A. In the latter well, the above mentioned taxa are accompanied by numerous other species including Belonechitina micracantha typica, Cyathochitina latipatagium, D. (Pseudodesmochitina) minor f. typica, Spinachitina bulmani, Conochitina elegans, Belonechitina capitata and a new species called here Spinachitina sp. A. In addition, two other taxa that are present, Acanthochitina barbata and Lagenochitina baltica have wide geographical distri- 
butions but ranges which are restricted to the OnnianPusgillian-Early Cautleyan (Jenkins, 1967; Laufeld, 1967; Achab, 1977 a, b, 1978 a, b; Nolvak, 1980; Grahn, 1982). The Late Ordovician Chitinozoan assemblages from Well J1-81A display strong affinities with those described in the Upper Ktaoua Formation (Morocco) which is referred to the Pusgillian-Cautleyan (Elaouad-Debbaj, 1984), and with those from the middle part of the Vauréal Formation referred to the Pusgillian (Achab, 1977a). However, the apparent absence of the genus Hercochitina from the Libyan material should be noted. The samples from Well J1-81 A, especially the youngest, are of Early Ashgill age.

The Ordovician Chitinozoan assemblages from Well E1-81 are very close to those described by Bouché (1965) from the Kourneida Well (Djado, Niger) which also appear to be of Ashgill age. A more precise stratigraphical assignment is difficult because of the lack of good stratigraphical control on the Djado material.

\section{REFERENCES}

Achab, A. 1977a. Les chitinozoaires de la zone à Dicellograptus complanatus. Formation de Vauréal, Ordovicien supérieur, Ile d'Anticosti, Québec. Can. J. Earth Sci., 14, 413-425.

Achab, A. 1977b. Les chitinozoaires de la zone à Climacograptus prominens elongatus de la Formation de Vauréal (Ordovicien supérieur), Ile d'Anticosti, Québec. Can.J. Earth Sci., 14, 2193-2212.

Achab, A. 1978a. Sur quelques chitinozoaires de la Formation de Vauréal et de la Formation de Macasty (Ordovicien supérieur). Ile d'Anticosti, Québec, Canada. Rev. Palaeobot. Palynol., 25, 295-314.

Achab, A. 1978b. Les Chitinozoaires de l'Ordovicien supérieur, Formations de Vauréal et Ellis Bay de l'Ile Anticosti, Québec. Palinologia, num. ext. 1-19.

Bouché, P.M. 1965. Chitinozoaires du Silurien s.l. du Djado (Sahara nigérien). Rev. Micropal., 8, 151-164.

Colbath, G. K. 1979. Organic-walled microphytoplankton from the Eden Shale (Upper Ordovician), Indiana, USA. Palaeontographica Abt. B., 171, 1-38.

\section{Explanation of Plate 1}

Fig. 1. Actinotodissus cf. crassus Loeblich \& Tappan, 1978. E1-81, 2790-2840 ft., Slide 1, E40/3, × 400, AGC 1.

Fig. 2. A. cf. crassus Loeblich \& Tappan, 1978. E1-81, 2790-2840 ft., Slide 1, V38/2, × 400, AGC 2.

Fig. 3. Goniosphaeridium cf. conjunctum Kjellstrom, 1971. E1-81, 2790-2840 ft., Slide 1, P42/0, × 400, AGC 3.

Fig. 4. G. cf. conjunctum Kjellstrom, 1971. E1-81, 2790-2840, Slide 1, S33/2, × 400, AGC 4.

Fig. 5. Leiofusa sp. E1-81, 2562-2567 ft., Slide 1, R45/0, × 160, AGC 5.

Fig. 6. Dactylofusa spinata? (Staplin, Jansonius \& Pocock) Combaz, Lange \& Pansart, 1967. E1-81, 2790-2840 ft., Slide $1, \mathrm{C} 41 / 4, \times 400, \mathrm{AGC} 6$.

Fig. 7. Eupoikilofusa striata? (Staplin, Jansonius \& Pocock) Eisenack, Cramer \& Diez, 1976. E1-81, 2562-2567 ft., Slide 1, Q44/3, × 400, AGC 7.

Fig. 8. Peteinosphaeridium nudum (Eisenack) Eisenack, 1969. E1-81, 2790-2840 ft., Slide 1, R32/0, × 400, AGC 8.

Fig. 9. D. spinata? (Staplin, Jansonius \& Pocock) Combaz, Lange \& Pansart, 1967. E1-81, 2790-2840 ft., Slide 1, V37/0, × 400, AGC 9.

Fig. 10. P. nudum (Eisenack) Eisenack, 1969. E1-81, 2790-2840 ft., Slide 1, C33/1, × 400, AGC 10.

Fig. 11. Ordovicidium heteromorphicum? (Kjellstrom) Turner, 1984. E1-81, 2790-2840 ft., Slide 1, K37/0, × 400, AGC 11.

Fig. 12. D. spinata? (Staplin, Jansonius \& Pocock) Combaz, Lange \& Pansart, 1967. E1-81, 2790-2840 ft., Slide 1, $\mathrm{V} 37 / 0, \times 1000, \mathrm{AGC} 9$.

Fig. 13. O. heteromorphicum? (Kjellstrom) Turner, 1984. E1-81, 2790-2840 ft., Slide 1, F44/1, × 400, AGC 12.

Fig. 14. Baltisphaerosum christoferii (Kjellstrom) Turner, 1984. E1-81, 2790-2840 ft., Slide 1, P46/0, × 400, AGC 13.

Fig. 15. A. cf. crassus Loeblich \& Tappan, 1978. E1-81, 2790-2840 ft., Slide 1, E40/3, × 1000, AGC 1.

Fig. 16. B. christoferii (Kjellstrom) Turner, 1984. E1-81, 2790-2840 ft., Slide 1, N37/0, × 400, AGC 14.

Fig. 17. B. christoferii (Kjellstrom) Turner, 1984. E1-81, 2790-2840 ft., Slide 1, U37/3, × 400, AGC 15. 
S. G. Molyneux

Plate 1

Late Ordovician Acritarchs
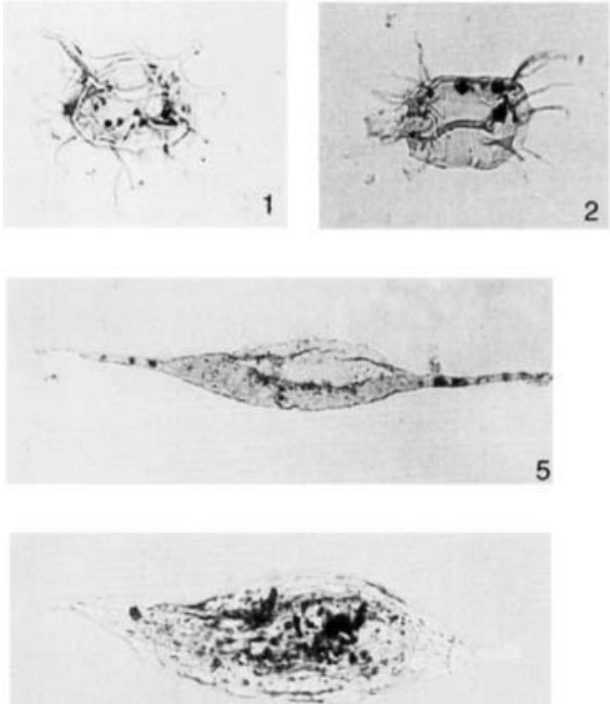

6
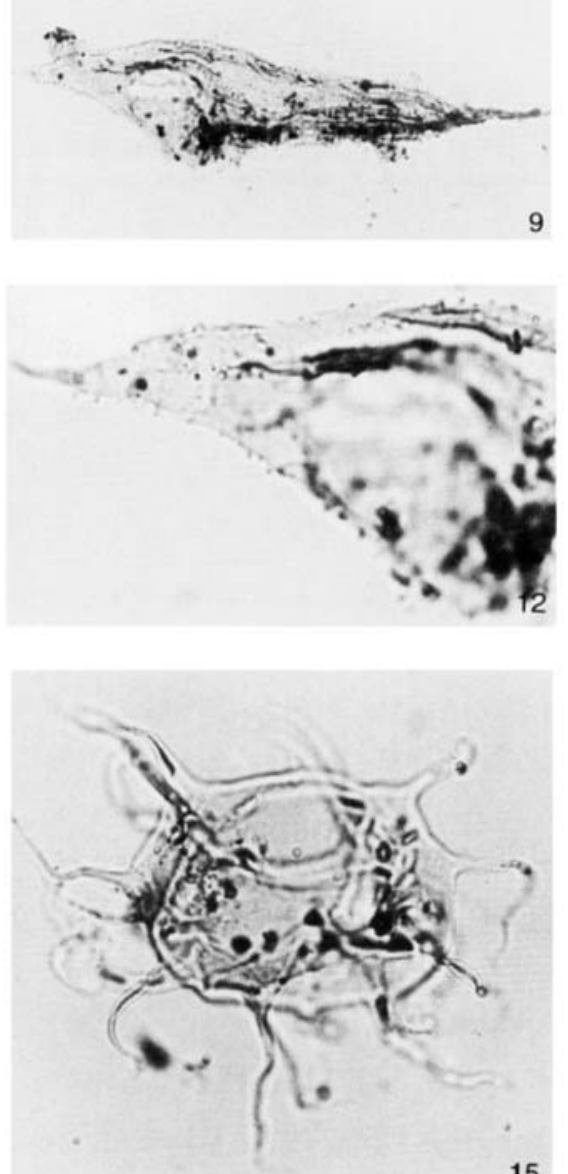
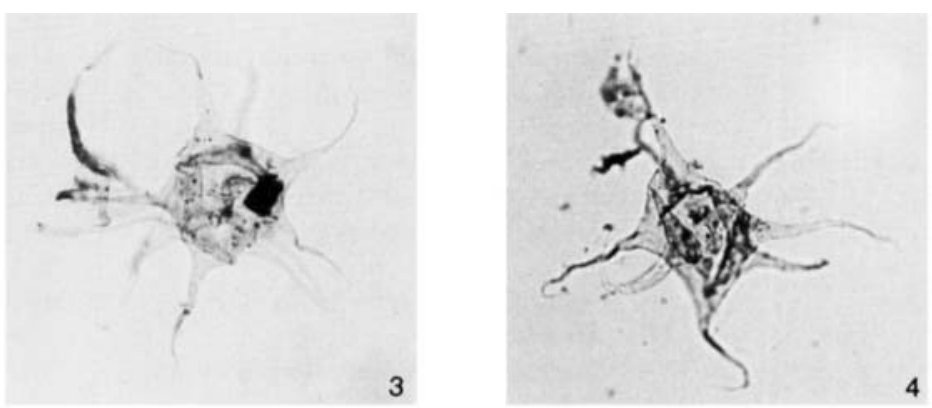

5
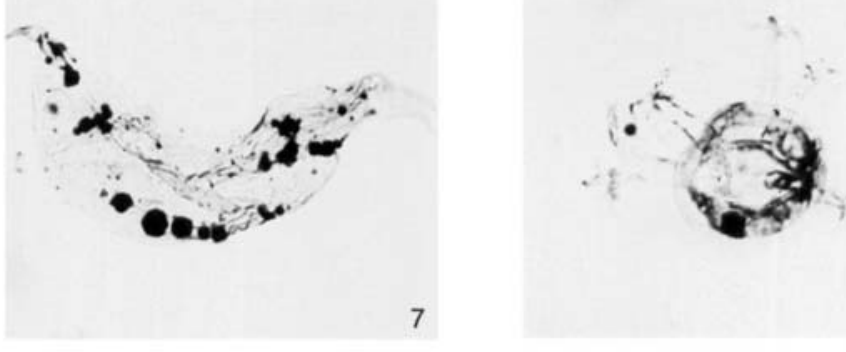

8
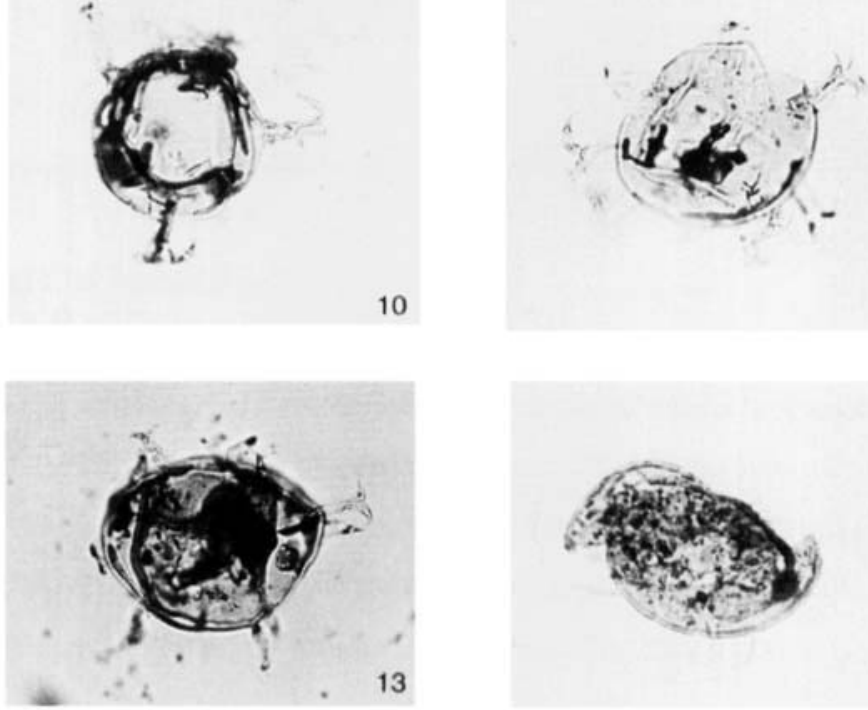

10

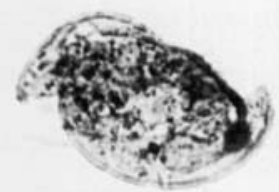

14
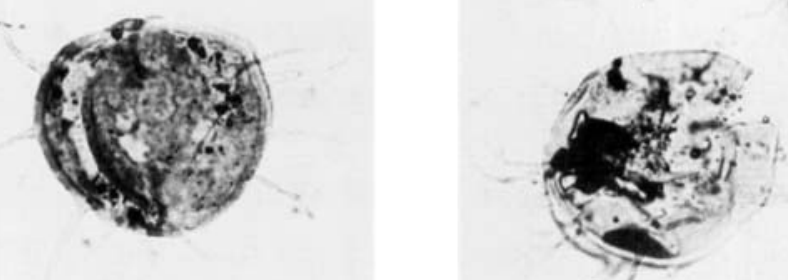
Cramer, F. H. \& Diez, M. d. C.R. 1979. Lower Palaeozoic acritarchs. Palinologia, 2, 17-160.

Elaouad-Debbaj, Z. 1984. Chitinozoaires ashgilliens de l'Anti-Atlas (Maroc). Geobios, 17 (1), 45-68.

Grahn, Y. 1982. Caradocian and Ashgillian Chitinozoa from the subsurface of Gotland. Sver. Geol. Unders. Ser.C, 788, $1-66$.

Jardiné, S., Combaz, A., Magloire, L., Peniguel, G. \& Vachey, G. 1974. Distribution stratigraphique des acritarches dans le Paléozoique du Sahara Algérien. Rev. Palaeobot. Palynol., 18, 99-129.

Jenkins, W.A.M. 1967. Ordovician Chitinozoa from Shropshire. Palaeontology, 10, 436-488.

Jenkins, W.A.M. 1970. Chitinozoa from the Ordovician Sylvan Shale of the Arbuckle Mountains, Oklahoma.Palaeontology, 13 (2), 261-288.

Kjellstrom, G. 1971. Ordovician microplankton (baltisphaerids) from the Grötlingbo Borehole No. 1 in Gotland, Sweden. Sver.Geol.Unders. Series C, 655, 1-75.
Laufeld, S. 1967. Caradocian Chitinozoa from Dalarna, Sweden. Geol. För. Stockh. Förh., 89, 275-349.

Loeblich, A.R. Jr. 1970 (for 1969). Morphology, ultrastructure and distribution of Paleozoic acritarchs. Proc. N. Amer. Paleontol. Conv. Chicago 1969 Part G, 705-788.

Loeblich, A.R. Jr., \& Tappan, H. 1969. Acritarch excystment and surface ultrastructure with descriptions of some Ordovician taxa. Rev. Esp. Micropaleontol., 1, 45-57.

Loeblich, A.R. Jr., \& Tappan, H. 1978. Some Middle and Late Ordovician microphytoplankton from central North America. J. Paleontol., 52, 1233-1287.

Nolvak, J. 1980. Chitinozoans in biostratigraphy of the Northern East Baltic Ashgillian. A preliminary report, Acta Palaeont. Polonica., 25, 253-260.

Turner, R.E. 1984. Acritarchs from the type area of the Ordovician Caradoc Series, Shropshire, England. Palaeontographica, Abt. B., 190, 87-157.

\section{Explanation of Plate 2}

Fig. 1. Leiofusa sp. E1-81, 2520-2550 ft., Slide 1, M30/1, × 400, AGC 16.

Fig. 2. Baltisphaeridium longispinosum delicatum Turner, 1984. E1-81, 2562-2567 ft., Slide 1, N44/3, × 400, AGC 17.

Fig. 3. B. longispinosum delicatum Turner, 1984. E1-81, 2790-2840 ft., Slide 1, H50/1, × 400, AGC 18.

Fig. 4. Baltisphaeridium sp. E1-81, 2562-2567 ft., Slide 1, D40/0, × 400, AGC 19.

Fig. 5. B. longispinosum delicatum Turner, 1984. E1-81, 2562-2567 ft., Slide 1, G34/3, × 400, AGC 20.

Fig. 6. Aremoricanium syringosagis? Loeblich \& MacAdam, 1971. E1-81, 2552-2557 ft., Slide 1, J46/4, $\times 400$, AGC 21.

Fig. 7. Orthosphaeridium cf. chondrododora Loeblich \& Tappan, 1971. E1-81, 2790-2840 ft., Slide 1, T42/2, × 400, AGC 22.

Fig. 8. Baltisphaerosum? sp. E1-81, 2790-2840 ft., Slide 1, M31/4, × 400, AGC 23.

Fig. 9. ?Baltisphaerosum bystrentos (Loeblich \& Tappan) Turner, 1984. E1-81, 2790-2840 ft., Slide 1, Q44/0, × 400, AGC 24.

Fig. 10. Baltisphaerosum? sp. E1-81, 2790-2840 ft., Slide 1, M31/4, × 1000, AGC 23.

Fig. 11. Navifusa similis? (Eisenack) Turner, 1984. E1-81, 2552-2557 ft., Slide 1, B32/0, × 1000, AGC 25.

Fig. 12. Eupoikilofusa striata? (Staplin, Jansonius \& Pocock) Eisenack, Cramer \& Diez, 1976. E1-81, 2552-2557 ft., Slide 1, D41/0, × 400, AGC 26.

Fig. 13. Leiofusa sp. E1-81, 2552-2557 ft., Slide 1, C30/0, × 160, AGC 27. 
S. G. Molyneux

Plate 2

Late Ordovician Acritarchs
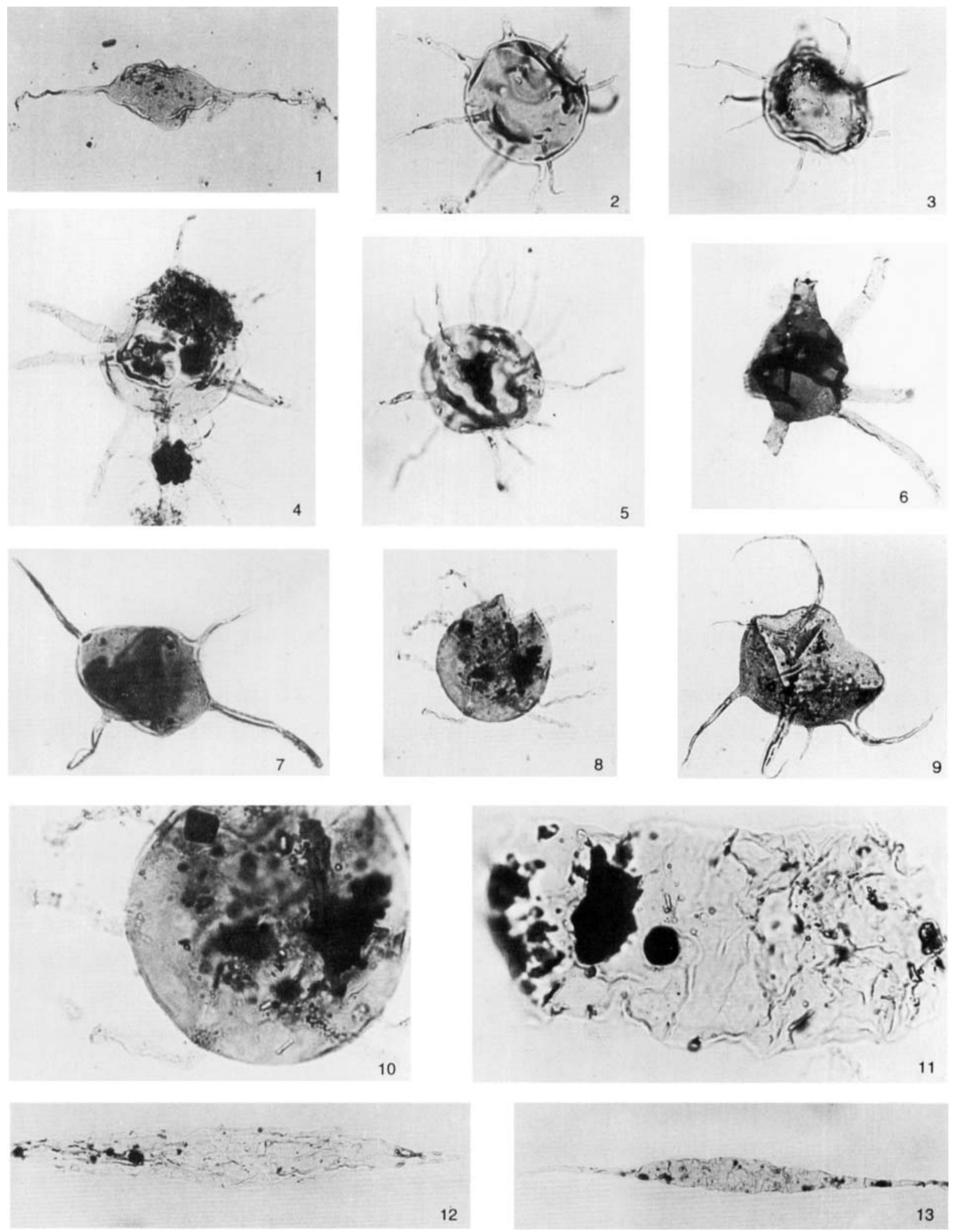


\section{Explanation of Plate 3}

Fig. 1. Veryhachium trispinosum (Eisenack) Deunff 1954 ex Downie, 1959. E1-81, 2520-2550 ft., Slide 1, U35/0, $\times 400$, AGC 28 .

Fig. 2. Veryhachium irroratum Loeblich \& Tappan, 1969. E1-81, 2552-2557 ft., Slide 1, P31/2, × 400, AGC 29.

Fig. 3. Villosacapsula setosapellicula (Loeblich) Leoblich \& Tappan, 1976. E1-81, 2520-2550 ft., Slide 1, U38/0, × 400, AGC 30.

Fig. 4. Veryhachium subglobosum Jardiné, Combaz, Peniguel \& Vachey, 1974. E1-81, 2790-2840 ft., Slide 1, U33/2, $\times 400$ AGC 31 .

Fig. 5. V. subglobosum Jardiné et al., 1974. E1-81, 2790-2840ft., Slide 1, D33/0, × 400, AGC 32.

Fig. 6. V. subglobosum Jardiné et al., 1974. E1-81, 2790-2840 ft., Slide 1, M39/2, × 400, AGC 33.

Fig. 7. Veryhachium cf. reductum (Deunff) Jekhowsky, 1961. E1-81, 2790-2840 ft., Slide 1, V43/3, × 400, AGC 34.

Fig. 8. $V$. cf. reductum (Deunff) Jekhowsky, 1961. E1-81, 2790-2840 ft., Slide 1, V43/3, × 1000, AGC 34.

Fig. 9. V. cf. reductum (Deunff) Jekhowsky, 1961. E1-81, 2790-2840 ft., Slide 1, H42/0, × 1000, AGC 35.

Fig. 10. V. cf. reductum (Deunff) Jekhowsky, 1961. E1-81, 2790-2840 ft., Slide 1, H42/0, $\times 1000$, phase contrast, AGC 35.

Fig. 11. V. setosapellicula (Loeblich) Loeblich \& Tappan, 1976. E1-81, 2552-2557 ft., Slide 1, F40/4, $\times 1000$, AGC 36.

Fig. 12. V. irroratum Loeblich \& Tappan, 1969. E1-81, 2552-2557 ft., Slide 1, P31/2, × 1000, AGC 29.

Fig. 13. V. irroratum Loeblich \& Tappan, 1969. E1-81, 2552-2557 ft., Slide 1, T46/4, × 1000, AGC 37.

Fig. 14. V. setosapellicula (Loeblich) Loeblich \& Tappan, 1976. E1-81, 2552-2557 ft., Slide 1, F40/4, $\times 1000$, phase contrast, AGC 36. 
Late Ordovician Acritarchs
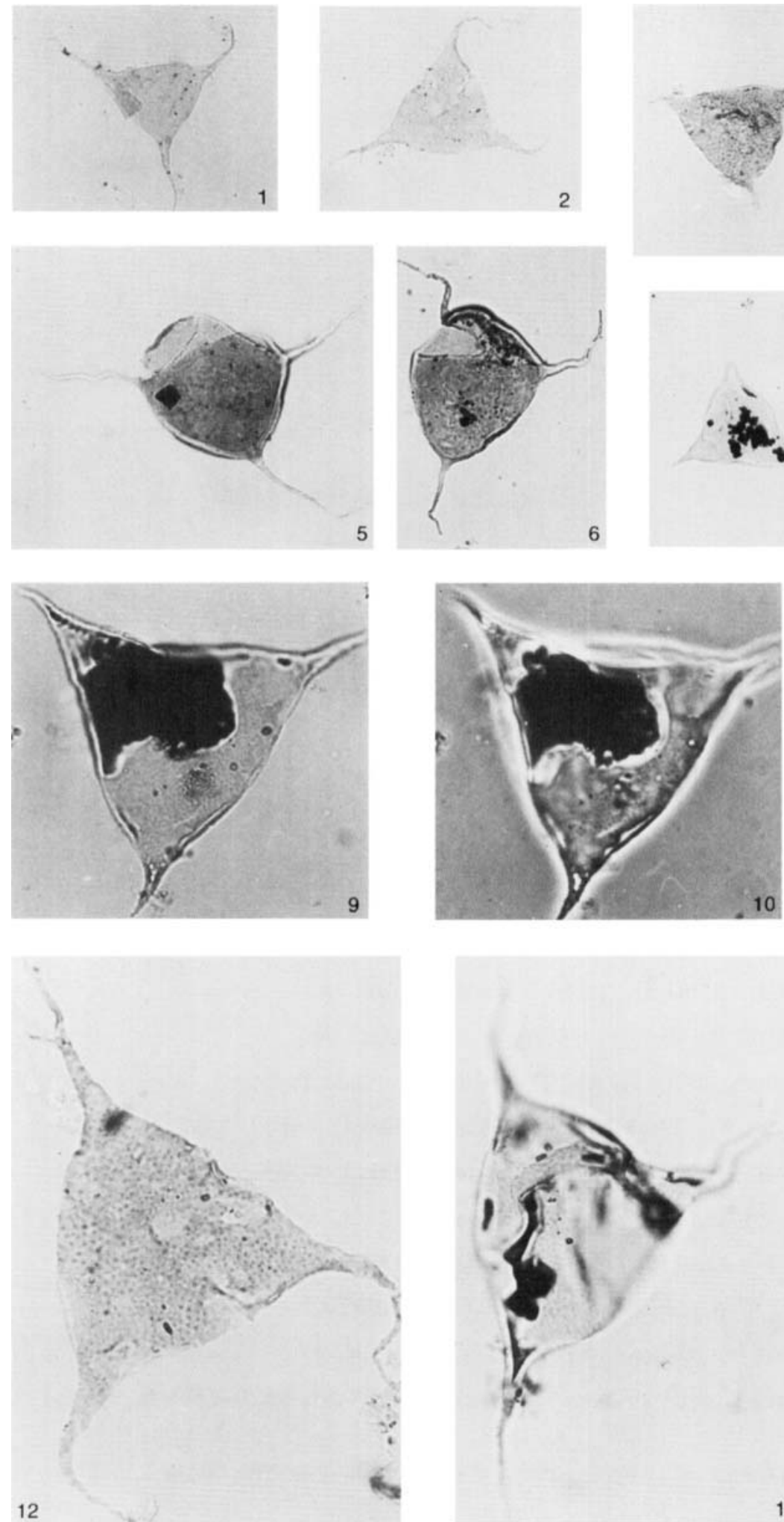

S. G. Molyneux
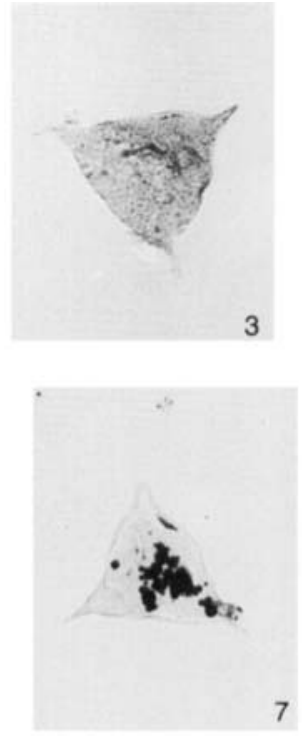

Plate 3
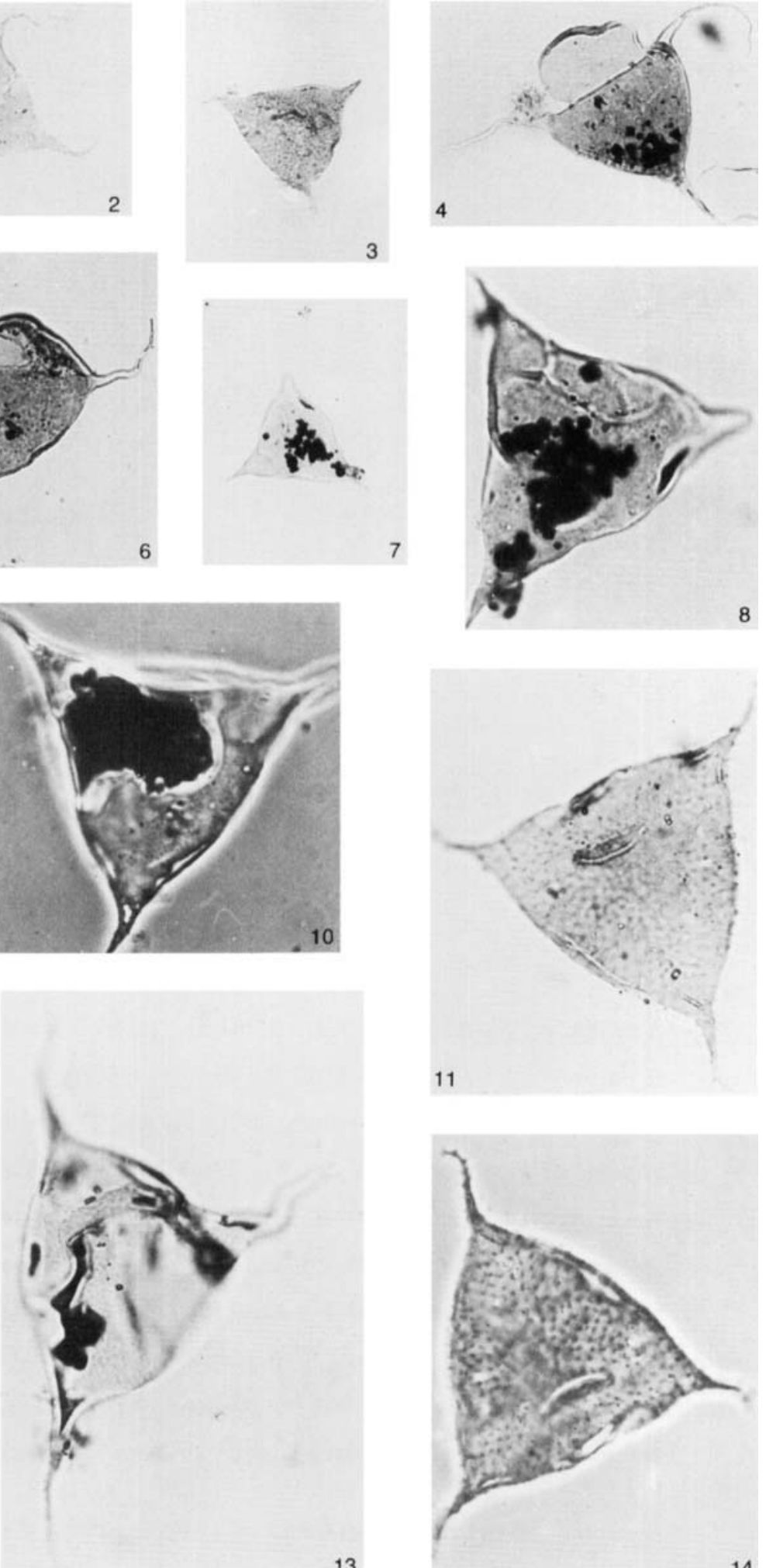

13

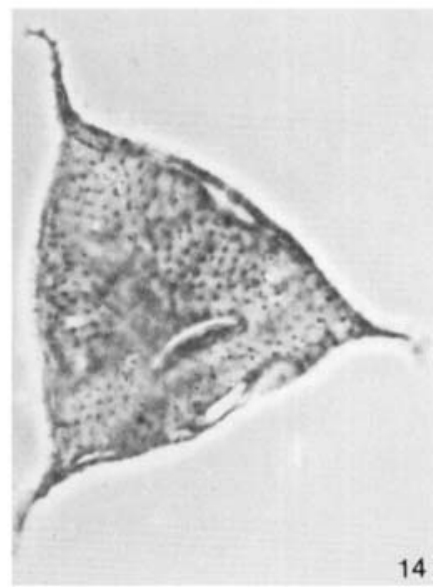




\section{Explanation of Plate 4}

Fig. 1. Striatotheca sp. A. E1-81, 2562-2567 ft., Slide 1, 035/3, × 400, AGC 38.

Fig. 2. Striatotheca sp. A. E1-81, 2552-2557 ft., Slide 1, U33/0, × 400, AGC 39.

Fig. 3. Veryhachium oklahomense ? Loeblich, 1970. E1-81, 2790-2840 ft., Slide 1, R45/3, × 400, AGC 40.

Fig. 4. V. oklahomense? Loeblich, 1970. E1-81, 2790-2840 ft., Slide 1, H39/4, × 400, AGC 41.

Fig. 5. V. oklahomense? Loeblich, 1970. E1-81, 2552-2557 ft., Slide 1, U38/3, × 400, AGC 42.

Fig. 6. V. oklahomense? Loeblich, 1970. E1-81, 2552-2557 ft., Slide 1, U38/3, × 1000, AGC 42.

Fig. 7. V. oklahomense? Loeblich, 1970. E1-81, 2790-2840 ft., Slide 1, H39/4, × 1000, AGC 41.

Fig. 8. Striatotheca sp. A. E1-81, 2552-2557 ft., Slide 1, T39/4, $\times 1000$, AGC 43.

Fig. 9. Striatotheca sp. A. E1-81, 2552-2557 ft., Slide 1, U33/0, × 1000, AGC 39.

Fig. 10. Veryhachium cf. lairdii (Deflandre) Deunff 1959 ex Downie, 1959. E1-81, 2520-2550 ft., Slide 1, U35/0, $\times 1000$, AGC 44 .

Fig. 11. V. cf. lairdii (Deflandre) Deunff 1959 ex Downie, 1959. E1-81, 2790-2840 ft., Slide 1, V37/4, × 1000, AGC 45.

Fig. 12. Striatotheca sp. A. E1-81, 2562-2567 ft., Slide 1, 035/3, × 1000, AGC 38. 
S. G. Molyneux

Late Ordovician Acritarchs
Plate 4
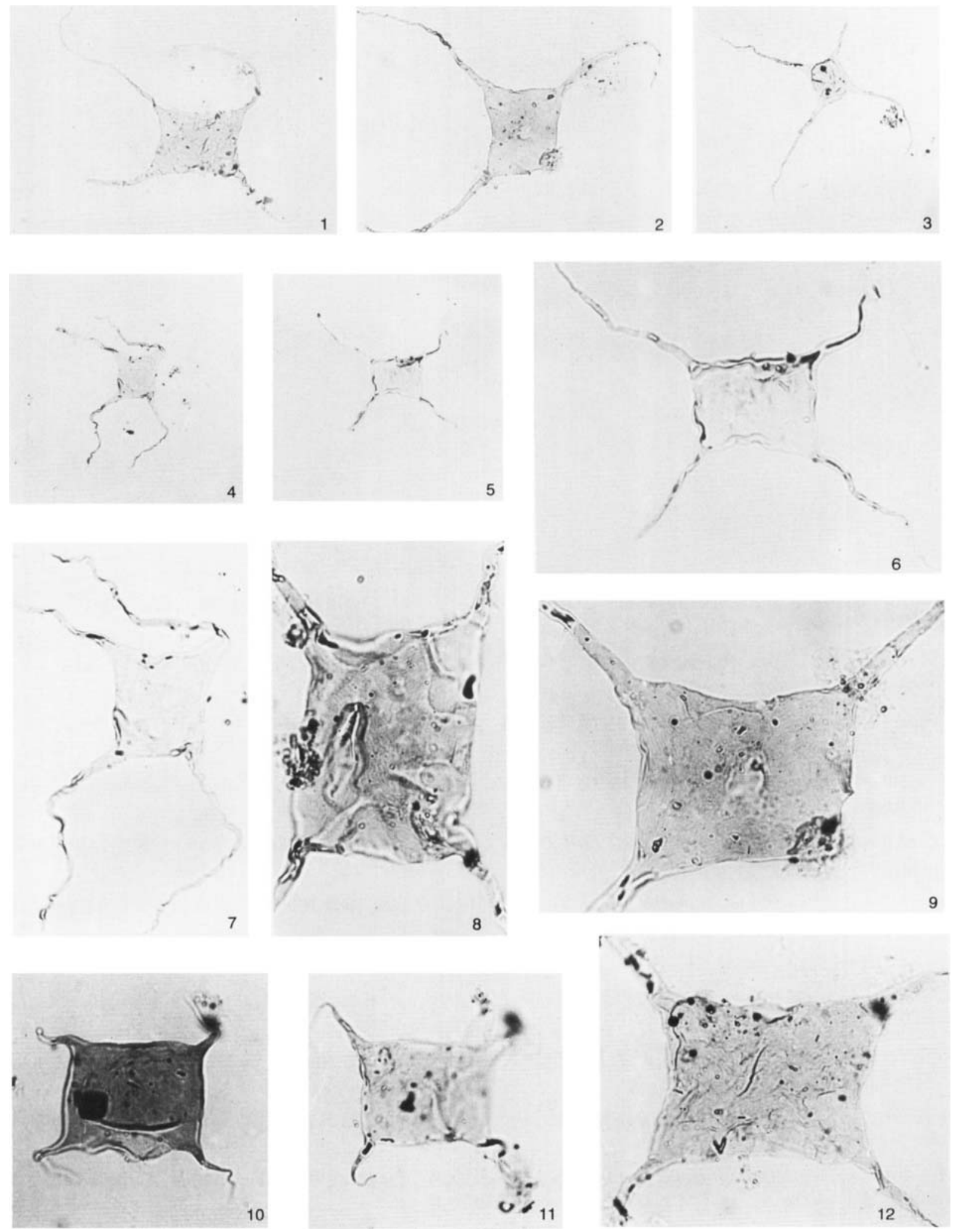


\section{Explanation of Plate 5}

Fig. 1 a-b. Spinachitina bulmani (Jansonius, 1964). J1-81 A, 12800-12850 ft., Slide 2, 035, 1a : × 250; 1 b : × 1250 , AGC 46 (Caradoc-Ashgill).

Fig. 2. Plectochitina sylvanica (Jenkins, 1970). J1-81 A, 12950-13000 ft., Slide 1, 035/4, × 400, AGC 47. (Early Ashgill).

Fig. 3. Ancyrochitina cf. merga Jenkins, 1970. J1-81A, 12950-13000 ft., Slide 1, P38/4, × 300, AGC 48. (Early Ashgill for A. merga).

Fig. 4. Plectochitina sylvanica (Jenkins, 1970). J1-81 A, 12800-12850 ft., Slide 2, M35, × 300, AGC 49. (Early Ashgill).

Fig. 5. Plectochitina sylvanica ? (Jenkins, 1970). J1-81 A, 12800-12850 ft., Slide 2, P35, × 300, AGC 50. (Early Ashgill).

Fig. 6.Plectochitina sylvanica (Jenkins, 1970). J1-81 A, 12950-13000 ft., Slide 1, N38, × 400, AGC 51. (Early Ashgill).

Fig. 7. Tanuchitina bergstroemi Laufeld, 1967. J1-81 A, 12950-13000 ft., Slide 1, 039/4, × 150, AGC 52. (Latest Caradoc-Ashgill).

Fig. 8. Plectochitina spongiosa (Achab, 1977b). J1-81 A, 12950-13000 ft., Slide 1, 036/3, $\times 300$, AGC 53. (Ashgill Early Llandovery?).

Fig. 9. Plectochitina spongiosa (Achab, 1977b). J1-81 A, 12950-13000 ft., Slide 1, P41/2, × 300, AGC 54. (Ashgill Early Llandovery?).

Fig. 10. Belonechitina micracantha typica (Eisenack, 1965). J1-81 A, 12950-13000 ft., Slide 1, N37, × 300, AGC 55. (Late Arenig - Ashgill).

Fig. 11 a-b. Belonechitina micracantha typica (Eisenack, 1965). J1-81 A, 12950-13000 ft., Slide 1, P37/2, 11 a : $\times 200 ; 11 \mathrm{~b}: \times 1000$, AGC 56. (Late Arenig-Ashgill).

Fig. 12 a-b. Spinachitina sp. A. J1-81 A, 12950-13000 ft., Slide 1, P41/3, 12 a : × 300; 12 b : × 1000, AGC 57. 
F. Paris

Ashgill Chitinozoans

Plate 5

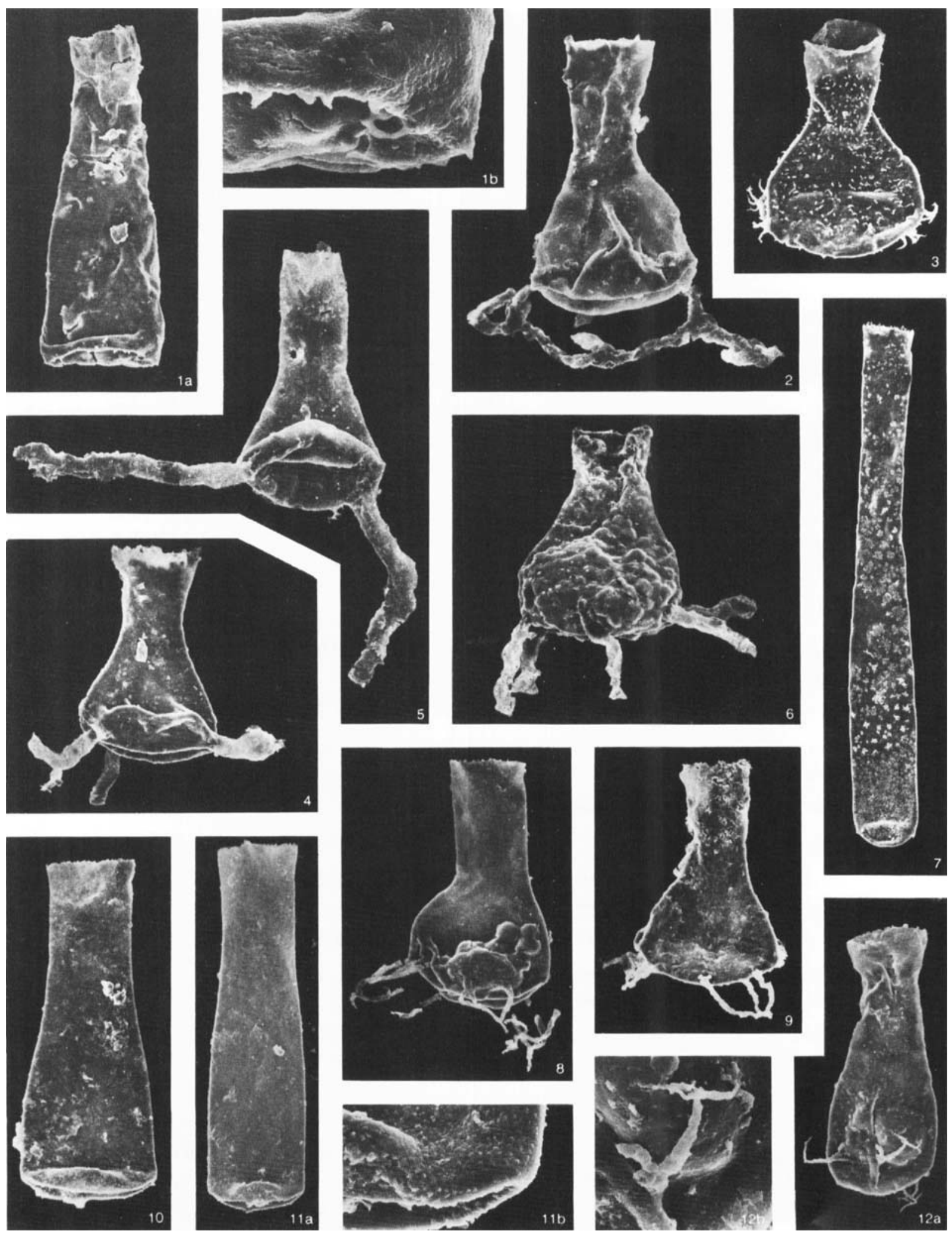




\section{Explanation of Plate 6}

Fig. 1 a,b. Acanthochitina barbata Eisenack, 1931. J1-81 A, 12150-12360 ft., Slide 3, R46/1, 1a : × 150; 1b: × 500, AGC 58. (Latest Caradoc - Early Ashgill).

Fig. 2. Spinachitina sp. A. J1-81 A, 12150-12360 ft., Slide 3, U44/1, × 300, AGC 59.

Fig. 3. Belonechitina capitata (Eisenack, 1962). J1-81 A, 12150-12360 ft., Slide 3, W43, × 150, AGC 60. (Late Llanvirn - Early Ashgill).

Fig. 4. Belonechitina sp. aff. schopfi (Taugourdeau, 1965). J1-81 A, 12150-12360 ft., Slide 3, V45/4, × 250, AGC 61 .

Fig. 5. ? Plectochitina sp. J1-81 A, 12150-12360 ft. Slide 3, U45/1, × 300, AGC 62 .

Fig. 6 a,b. Calpichitina (Calpichitina) lenticularis (Bouché, 1965). J1-81 A, 12150-12360 ft., Slide 3, T46/1, 6a : $\times 300 ; 6 \mathrm{~b}: \times 1250$, AGC 63. ("Middle" Caradoc - Ashgill).

Fig. 7. Tanuchitina anticostiensis (Achab, 1977a). E1-81, $2572 \mathrm{ft}$., Slide 5, P36, $\times$ 400, AGC 64. (Ashgill).

Fig. 8. Desmochitina (Pseudodesmochitina) minor forma typica Eisenack, 1958. J1-81 A, 12150-12360 ft., Slide 3, R45/4, × 300, AGC 65. (Llanvirn - Ashgill).

Fig. 9 Armoricochitina nigerica (Bouché, 1965). E1-81, 2572 ft., Slide 5, 036, × 200, AGC 66. (Late Caradoc Ashgill).

Fig. 10. Enlargement of the granulose ornamentation of Armoricochitina nigerica (Bouché, 1965) represented pl. 7 , fig. 1. E1-81, 2252- 2574 ft., Slide 4, J/39, × 1000, AGC 69.

Fig. 11. C. (Calpichitina) lenticularis (Bouché, 1965). Aboral view, J1-81 A, 12150-12360 ft., Slide 3, S46/2, × 300, AGC 67. ("Middle" Caradoc - Ashgill).

Fig. 12. Ancyrochitina cf. merga Jenkins, 1970. J1-81 A, 12800-12850 ft., Slide 2, 039/2, × 300, AGC 68. (A. merga is reported in the Ashgill). 
Ashgill Chitinozoans
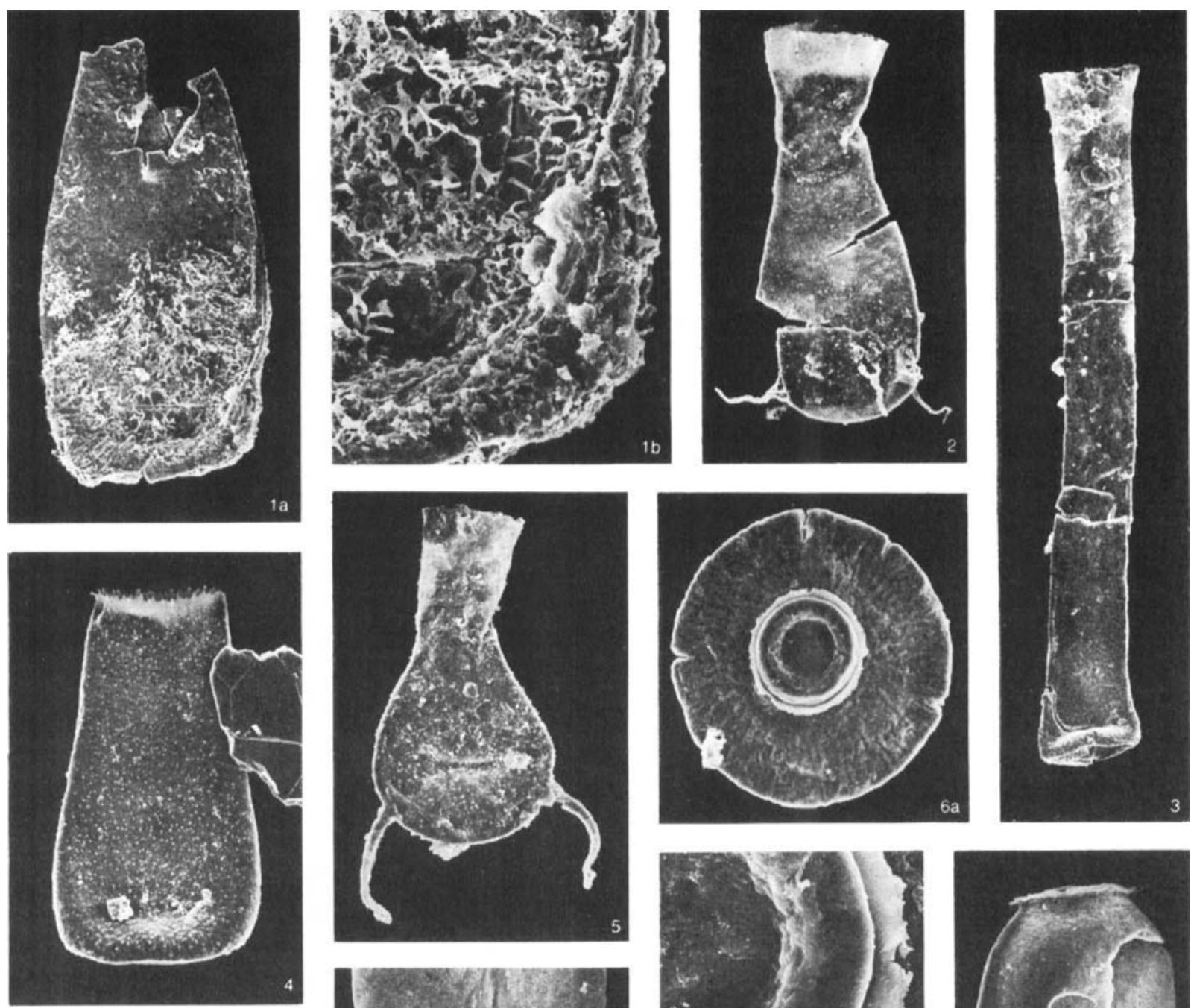

3
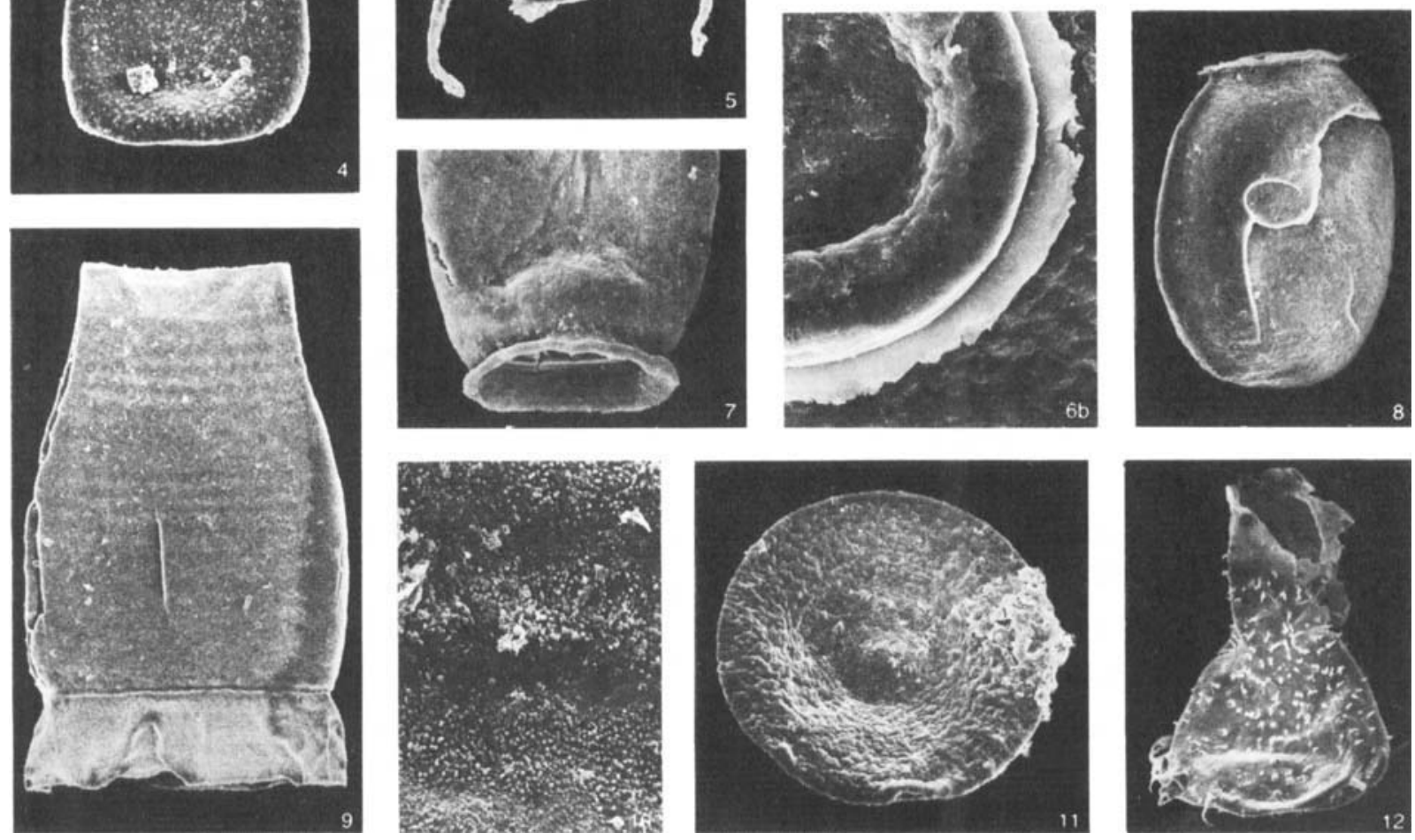


\section{Explanation of Plate 7}

Fig. 1.Armoricochitina nigerica (Bouché, 1965). E1-81, 2552-2574 ft., Slide 4, J39, × 250, AGC 69. (Late CaradocAshgill).

Fig. 2 a,b. Armoricochitina nigerica (Bouché, 1965). E1-81, 2552-2574 ft., Slide 4, J37/4, 2 a : × 300; 2b: × 750, AGC 70. (Late Caradoc - Ashgill).

Fig. 3. Armoricochitina nigerica (Bouché, 1965). E1-81, 2252-2574 ft., Slide 4, M40, $\times 1250$; details of the carina ; see the granulose ornamentation, AGC 71. (Late Caradoc - Ashgill).

Fig. 4. Lagenochitina baltica Eisenack, 1931. E1-81, 2572 ft., Slide 5, S37, × 300, AGC 72. (Late Caradoc-Early Ashgill).

Fig. 5. Ancyrochitina onniensis Jenkins, 1967. E1-81, 2552-2574 ft., Slide 4, P39, × 400, AGC 73. (Latest Caradoc (Onnian) - Ashgill).

Fig. 6 a,b. Tanuchitina bergstroemi Laufeld, 1967. E1-81, 2572 ft., Slide 5, S40/3, 6a : $\times 150 ; 6$ b : $\times 750$, AGC 74 . (Latest Caradoc - Ashgill).

Fig. 7. Belonechitina micracantha (Eisenack, 1931). E1-81, $2572 \mathrm{ft}$., Slide 5, 035/1, $\times 200$, AGC 75. (Ordovician).

Fig. 8 a,b. Belonechitina sp. A. E1-81, 2572 ft., Slide 5, R36, 8a : × 250;8b : × 1000, AGC 76.

Fig. 9. a,b. Spinachitina suecica (Laufeld, 1967). E1-81, 2572 ft., Slide 5, 035, 9a : $\times 400 ; 9 \mathrm{~b}: \times 1000$, AGC 77. (Caradoc).

Fig. 10. Calpichitina (Calpichitina) lenticularis (Bouché, 1965). E1-81, $2572 \mathrm{ft}$., Slide 5, N38/1, $\times 300$, AGC 78 . ("Middle" Caradoc - Ashgill). 
F. Paris

Late Caradoc - Ashgill Chitinozoans
Plate 7
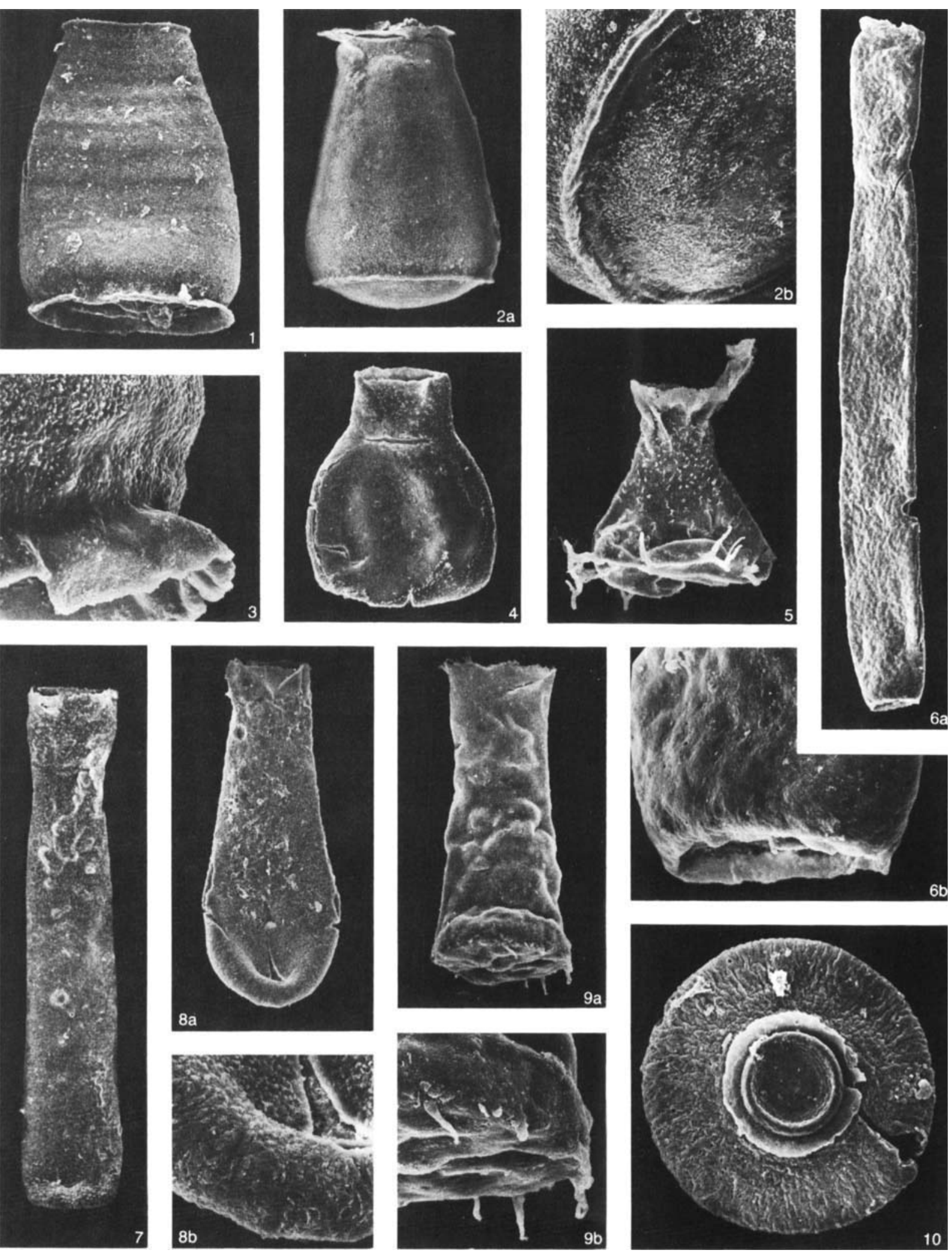